\title{
Zeremonien im Lebensablauf der Balinesen
}

\section{Einleitung und Problemstellung}

"Wo Sonne und Mond scheinen, wo sich Vulkane erheben und Wasser deren Flanken hinunterfließt, da ist auch Gott, der die Menschen segnet.» So lautet ein Satz aus dem Lontarbuch ${ }^{1}$ Tantu Pagelaran, welcher die Geborgenheit der Balinesen in ihrem Glauben und in ihrem Lebensraum ausdrückt. Unzweifelhaft ist der Begriff «Heimat» auch für Balinesen von ortsgebundener Identifikation geprägt. "Heimat» ist aber mehr als territoriale Bodenverhaftung, verweist doch der Begriff vor allem auf das Lernfeld der Sozialisation und bestimmte Qualitäten zwischenmenschlicher Beziehungen, die zu erarbeiten gerade auf Bali interessant sind.

Wie groß die Gegensätze zwischen Göttern und Balireisenden auch sein mögen: In einem kommen sie einander recht nahe. Denn beide erfreuen sich an farbenfrohen Opfergaben, und beide wissen Feste und Musik, Tänze und Schauspiele zu schätzen. Da Balinesen ihre Empfindungen weniger durch Worte als vielmehr durch Handlungen ausdrücken, fällt es Inselbesuchern leicht, Äußerlichkeiten (sekala) verschiedener Zeremonien in Bildern festzuhalten, wobei sich die Wahrnehmung freilich oft nur auf Teilbereiche eines in sich geschlossenen Zyklus beschränkt und der Dokumentierung eines exotischen Spektakels dient, das «Unsichtbare» (niskala) aber außer acht läßt.

Eine Analyse fundamentaler Prinzipien fällt deshalb nicht immer leicht, weil auf Bali autochthone Denkweisen und indisch-hinduistische Argumentationen sowohl nebeneinander existieren als sich auch vermengen. Letzteres ist vor allem dann der Fall, wenn balinesische Brahmanenpriester (padanda) und Adatgelehrte ${ }^{2}$ zur wissenschaftlichen Deutung Zugriff auf die indische Philosophie nehmen, mit anderen Worten einheimisches Handeln über einen fremden Raster zu begründen bestrebt sind. Zusätzliche Komplikationen ergeben sich aus der Tatsache, daß direkter Beobachtung zugängliche Phänomene sowohl aufgrund der lokalen Adatvielfalt als auch infolge unterschiedlicher Kastenzugehörigkeit variieren. Fragen nach der ursächlichen Begründung ihres Handelns können oft deshalb nicht beantwortet werden, weil Balinesen das tun, was sie als adatkonform erachten, ohne immer den Sinngehalt ihrer Aktivitäten zu hinterfragen. Grundsätzlich gilt, daß sich die tief in der Religion verankerte Kultur durch ein ausgeprägtes Streben nach Harmonisierung von Gegensätzlichkeiten auszeichnet und auf das Ausbalancieren von göttlichen und chthonischen Kräften abzielt.

Die Gesamtheit balinesischer Zeremonien wird in fünf Kategorien (panca yadnya) gegliedert:

1. Rituale zur Verehrung Gottes, seiner verschiedenen uranischen Manifestationen und der vergotteten Ahnen (dewa yadnya);

2. Zeremonien zur Beschwichtigung chthonischer Aspekte Gottes, zur Besänftigung von Dämonen und zur Vertreibung übelstiftender Kräfte, welche die Individuen sowie deren natürliche Umwelt und soziales Umfeld bedrohen (buta yadnya);

3. Zeremonien im Lebensablauf eines Menschen (manusa yadnya);

4. Zeremonien für Körper und Seele Verstorbener (pitra yadnya);

5. Zeremonien zur Priesterweihe (resi yadnya).

Im vorliegenden Aufsatz werden die manusa yadnya vorgestellt. Es soll u. a. aufgezeigt werden, daß Balinesen im menschlichen Körper (buana alit: "Kleine Welt») das Ebenbild des Universums bzw. der Welt (buana agung: "Grosse Welt») erkennen, somit die Synthese von Makround Mikrokosmos die Denkweise Einheimischer prägt, und die Kosmogenie und Schöpfung in Beziehung zu den vier die Menschen begleitenden «Geschwistern» gesetzt wird (HOOYKAAS 1974: 171; LEEMANN 1976: 27-65; WECK 1976: 139).

\section{Zeremonien im Lebensablauf}

\subsection{Ziele}

Upacara manusa yadnya sind Zeremonien im Ablauf eines Menschenlebens. Sie zielen auf körperliche Reinigung und geistige Läuterung ab und sollen die Individuen mit spiritueller Kraft erfüllen, so daß sie während ihres Gefahren und Anfechtungen ausgesetzten irdischen Daseins erfolgreich bestehen können. Nur ganz wenige Personen haben dank Askese und disziplinierter Meditation die hohe Stufe erreicht, die es ihnen erlaubt, sich aus eige-

Albert Leemann, Prof., Dr., Geographisches Institut der Universität Zürich-Irchel, Winterthurerstr. 190, 8057 Zürich 
ner Geisteskraft zu purifizieren. Der Großteil der Gläubigen bedarf dafür des Beistandes von Mitmenschen und materieller Symbole, so der Durchführung von Zeremonien (upacara) und - untrennbar damit verbunden - der Darreichung von Opfergaben (upakara). Gemäß hinduistischen Prinzipien ist die lebenslange Reinigung von Körper und Geist Voraussetzung zur Verinnerlichung göttlicher Inspiration, die den Menschen zu rechtem Denken, Sprechen und Handeln führt, sein karma (Gesetz der Tat) verbessert und ihm eine reine Seele gewährt, so daß diese nach dem Tod den Weg zu ihrem Ursprung findet. In ihrem jetzigen Leben tragen Wiedergeborene die Konsequenzen ihres Handelns aus früheren Erdendasein. Die Reinkarnation bietet dem Menschen eine Chance zur Vervollkommnung, welche Voraussetzung zur Entbindung aus dem Kreislauf von Leben und Tod (moksa) ist (PUTRA 1974/2: 5; 1987: vi).

In den upacara manusa yadnya kann das Streben nach Reinheit sowohl anhand von Opfergaben als auch in der reichlichen Verwendung von Weihwasser (tirta) ${ }^{3}$ erkannt werden (vgl. dazu ausführlich PUTRA?: Upakara-Yadnya). Der Sinngehalt der Purifizierung und deren Abstufungen gehen aus folgendem Zitat hervor:

«Der Körper des Menschen wird mit Wasser gereinigt, seine Gedanken durch Aufrichtigkeit, sein Geist durch Wissen und Askese und sein Verstand durch Weisheit" (PUTRA 1974/2: 6 f.).

\subsection{Grundelemente}

Upacara manusa yadnya setzen sich aus vier eng miteinander verknüpften Elementen zusammen:

\section{(1) Upacara mabiakala}

Diese Zeremonie ist integraler Bestandteil aller fünf yadnya (siehe oben). Dabei werden vor der Kontaktnahme mit dem Allmächtigen (bzw. dessen uranischen Manifestationen) den Repräsentanten der chthonischen Sphäre Opfergaben (biakala) dargeboten. Ziel dieser Handlung ist die Zufriedenstellung der Dämonen (buta kala), die nach entsprechender Würdigung nun nicht mehr als Störefriede auftreten, sondern den Versammelten helfen und Glück bringen. Mit entsprechenden Handgesten vom Körper weg (ngayab) nach hinten weisen die Gläubigen den buta kala den Weg vom Platz des gottbezogenen $\mathrm{Ri}$ tuals an ihren Ursprungsort zurück.

\section{(2) Upacara malukat und majaya-jaya}

Zielsetzung dieser Handlungen ist die Läuterung von Körper und Geist. Während beim malukat Weihwasser auf Opfergaben und Personen gesprenkelt wird, sind es beim majaya-jaya heilige Formeln (mantra), Weihrauch, Lämpchen mit brennendem Docht und Priesterglocken, die zur inneren und äußeren Stärkung beitragen sollen.

\section{(3) Upacara ngayab/natab}

Die hier präsentierten Opferarrangements dienen der Huldigungsbezeugung. Während beim ngayab banten biakala (vgl. 1) zur Wegweisung chthonischer Kräfte die Hände vom Körper weg nach hinten geschwenkt werden, ist es im Falle Gott geweihter ayaban-Opfer so, daß die
Gläubigen ihre Hände von der Brust weg nach vorn bewegen, um anzudeuten, daß sie die Essenz ihrer Präsente Gott zuzufächeln bestrebt sind. Bei tataban-Gaben sind die Gesten gerade umgekehrt: Der Feiernde schwenkt die Hände gegen seine Brust zu (natab). Damit drückt eine Person ihr Verlangen darnach aus, daß der Allmächtige sie nach der Beseelung der dargereichten Präsente segne, sich ihr zuwende und sie durchdringe. Die Gottmanifestationen werden gebeten, in spezifischen Körperorganen Platz zu nehmen (vgl. unten), um so das Handeln der Menschen von innen zu lenken.

\section{(4) Upacara muspa (beten)}

Falls die Betzeremonien bereits im Anschluß an das mabiakala stattfinden, bitten die Gläubigen, daß Gott Zeuge der Durchführung ihrer Feier im Lebenslauf sein möge. Muspa, die erst im Anschluß an das natab (vgl. oben) erfolgen, sind auf die Kontaktnahme mit dem Allmächtigen und den vergotteten Ahnen ausgerichtet. Abgeschlossen wird dieser Teil mit der Bitte um die Gewährung von Weihwasser und um die Zusprechung von wija (andere Orthographie: bija). Bei diesen handelt es sich um gewaschene, unversehrte Reiskörner, die in Sandelholzwasser oder in Wasser, versehen mit wohlriechenden Blütenblättern, eingeweicht worden sind. Weihwasser und Reiskörner werden dreimal verteilt und die wija zwischen die Augenbrauen sowie auf die Brust geklebt und auf die Zunge gelegt. In letzterem Fall werden sie anschließend verschluckt. Balinesen betrachten wija als Symbol vom Gott der Kleinkinder (Dewa Kumara) oder der Göttin der Fruchtbarkeit (Dewi Sri). Wie man sich von der Saat Früchte erhofft, so erwarten Gläubige von den genannten Gottmanifestationen, daß diese auf die feiernden Personen bzw. ihr Territorium ausstrahlen und somit Glück und Segen gewährleisten mögen. Nach Verabreichung der wija binden sich die Gefeierten weißes Garn ums Handgelenk oder legen solches über die als $\mathrm{Si}$ wa-Tor (Siwa duara) bezeichnete Fontanelle.

\subsection{Der menschliche Körper als Ausdruck des balinesischen Weltbildes}

Anhand der folgenden Schemata soll die balinesische Perzeption des menschlichen Körpers als Ebenbild des Universums und Südbalis verdeutlicht werden. Das Vierersystem nimmt Bezug auf die Achse bergwärts/meerwärts (in Südbali \pm Nord-Süd entsprechend) und der Richtung West-Ost. Falls die Windrose gemäß dem $45^{\circ}$ Prinzip unterteilt wird, resultiert entsprechend ein Achtersystem. Da die angesprochenen Richtungen immer von einem Mittelpunkt ausstrahlen, der die einzelnen Wertladungen integral zusammenfaßt, erweitern sich die jeweiligen Systeme um das Zentrum auf fünf bzw. neun. Die acht Manifestationen von Siwa als Allmächtigem werden als die "Acht Wächter der Richtungen» (astalokapala) bezeichnet. Man beachte, daß dem Tempelkomplex von Besakih eine Doppelfunktion zufällt: einerseits als Sitz des Wächters über die Richtung Nordosten und andererseits als Synthese der astalokapala. 
Wisnu (schwarz)<smiles>C=C</smiles>

Mahadewa (gelb)—— $\underset{\text { (füffarbig) }}{\text { Siwa }}$ Iswara (weiss)<smiles></smiles>

Fig. 1 Die Fünf Götter und die Fünf Farben (panca dewata und panca warna).

Galle (ampru)<smiles>C1CC1</smiles>

Nieren (ungsilan) — tumpuking ati* — Herz (papasuh)<smiles>CCCCCC</smiles>

Fig. 3 Sitzplatz der Fünf Götter im menschlichen Körper. * Tumpuking ati (bal.) bzw. tumpukan ati (ind.) beinhaltet - frei übersetzt - "oberhalb der Leber». Es wäre abzuklären, ob es sich bei dieser zentralen Stelle um den solar plexus handelt.
Pura Batur Ulun Danu

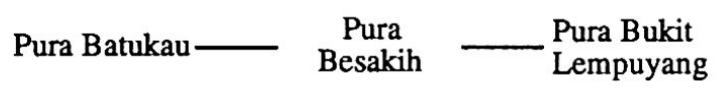

\section{$\left.\right|_{\text {Pura Andakasa }}$}

Fig. 2 Den Fünf Göttern zugesprochene Tempelstätten (pura).

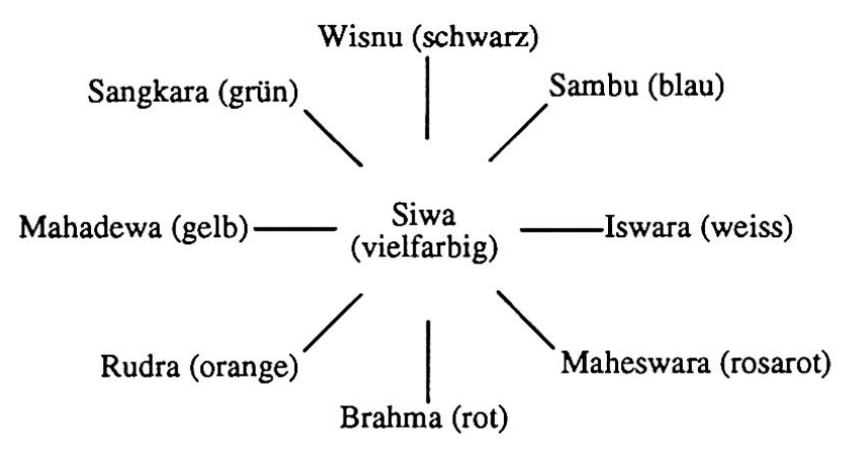

Fig. 4 Die Neun Götter und die Neun Farben (nawa dewata bzW. nawa sanga und nawa warna).

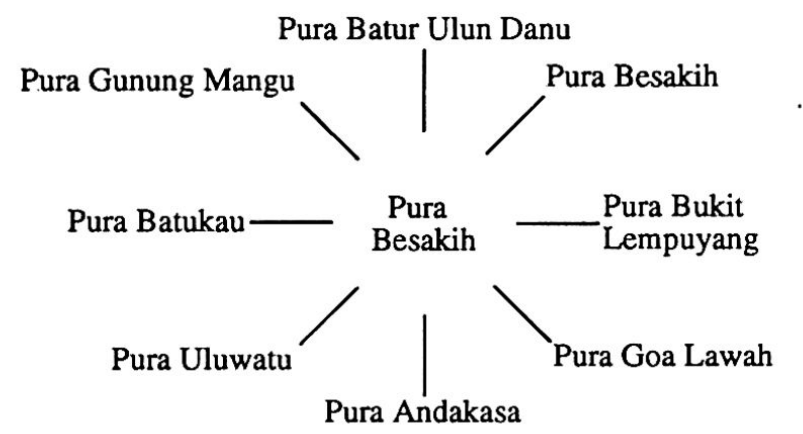

Fig. 5 Den Neun Göttern zugesprochene Tempelstätten.

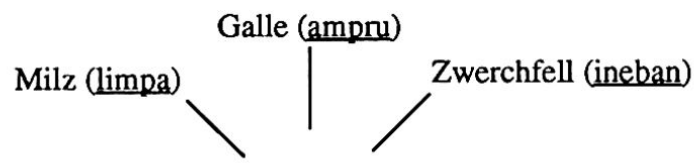

Nieren (ungsilan) — tumpuking ati _- Herz (papasuh)

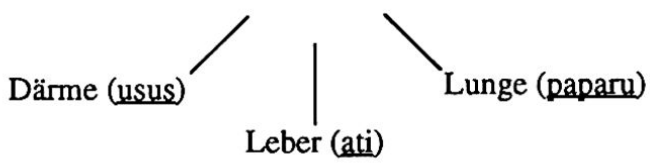

Fig. 6 Sitzplatz der Neun Götter im menschlichen Körper. 


\subsection{Ubersicht über die Zeremonien im Lebensablauf}

Manusa yadnya umfassen die Zeitspanne vom fötalen Zustand bis und mit der Verheiratung, die Phase also, in der die Eltern die Verantwortung für ihre Kinder zu tragen verpflichtet sind. Nach der Vermählung ihrer Söhne und Töchter sind Mutter und Vater der rituellen Sorgepflicht diesen gegenüber enthoben. Von diesem Zeitpunkt an obliegt der nachrückenden Generation die Fürsorge um ihre alternden Eltern und nach deren Ableben die Durchführung adatgemäßer Purifikationsrituale (pitra yadnya).

Eine vollständige Sequenz der rites de passage umfaßt folgende, meistens im Familienhof und in dessen Familientempel durchgeführte Zeremonien ${ }^{4}$ :
(1) Heirat (masakapan)
(2) Schwangerschaft (magedong-gedongan)
(3) Geburt
(4) Nabelschnurabfall (kepus puser)
(5) Zwölftagefeier (ngrorasin)
(6) 42-Tage-Feier (tutug kambuhan)
(7) Dreimonatsfeier (105-Tage-Feier: nelubulanin)
(8) erster Geburtstag (210-Tage-Feier: ngotonin)
(9) erstes Zahnen (ngempugin)
(10) erster Milchzahnausfall (maketus)
(11) durchlochen der Ohrläppchen
(12) erwachsen werden (munggah deha/teruni)
(13) Zahnfeilung (mapandes)

Aus dieser Vielfalt sollen im folgenden einige Zeremonien genauer beschrieben und deren Hintergründe erläutert werden.

\subsubsection{Heirat}

Mit der Vermählung bezeugen zwei Menschen vor Gott und ihrer Gemeinschaft, daß sie sich als Mann und Frau zu verbinden, in Zukunft einen gemeinsamen Haushalt zu führen und zusammen die Konsequenzen ihres Handelns zu tragen gewillt sind. Beim Eintritt in diesen neuen Lebensabschnitt ändern sich Rollen und Status insofern, als der Mann erst als Familienvorsteher vollberechtigtes Mitglied adatpolitischer Organisationen wird, die Frau gemäß patrilinearer Wohnfolgeordnung in den Hof ihres Mannes zieht und von nun an als Mitglied der dortigen Familie gilt. Auf welche Arten Ehen auch immer eingegangen werden - sei es auf Initiative der Eltern, sei es durch adatgemäße Fluchtheirat, bei der sich das Paar elterlicher Bevormundung entzieht - haben Eheschließungen wie andere Übergangszeremonien Purifikationscharakter. Doch nicht nur die Ehewilligen werden durch den Brahmanenpriester gereinigt, sondern auch die Wächter des väterlichen und mütterlichen "Samens» (kama jaya bzw. kama ratih). Ziel jeder balinesischen Heirat ist es nämlich, Kinder zu zeugen und - wenn immer möglich die Kontinuität der Patrilinie zu gewährleisten. Kinder sind nicht zuletzt auch deshalb erwünscht, weil sie dereinst nach dem Ableben ihrer Eltern die für die Befreiung deren Seelen und die Wiedergeburt unerläßlichen Toten- und Seelenreinigungsrituale durchführen können (RAMSEYER 1985: 203). Das Paar, dessen sexuelle Beziehungen durch die Heiratszeremonie legitimiert worden sind, erhofft sich von Purifikationsritualen für Spermien und Eizellen (madengen-dengen), daß übelwollende buta kala davon absehen, eine Befruchtung zu verhindern, und daß der sich entwickelnde Fötus zur Aufnahme einer reinen Seele fähig sei. Sollte eine Zeugung ohne vorheriges madengen-dengen-Zeremonial erfolgt sein, wird die resultierende Frucht als minderwertig betrachtet, und der Säugling läuft Gefahr, zum mißratenen Kind (rare dia-diu) zu werden.

Zwischen Familien verwandter oder sich verbunden fühlender Brautnehmer- und Brautgeberfamilien abgesprochene Heiraten gelten in den Augen vieler Balinesen zufriedenstellender als solche zwischen «Fremden». Denn Status- und Titelrivalitäten zwischen Kasten, deren Subkategorien, Geschlechter- und Tempelverbänden sind auf Bali oft Quell von Konflikten, die dann besonders gravierende Ausmaße annehmen können, wenn Heiraten als statusgefährdend bzw. als den Ahnen mißfallend perzeptiert werden (RAMSEYER 1985: 205 f.). Ursprünglichem adat gemäß intolerabel sind beispielsweise Heiraten hochkastiger Töchter mit kastenniedrigen Söhnen. Gerechtfertigt wird diese Norm mit der Metapher, daß der Same des Mannes - wie das Wasser - ausschließlich von "oben nach unten» fließen könne, mit anderen Worten, daß es einem Manne nur zustehe, Töchter gleichen oder niedrigeren Ranges zu ehelichen. Nichtadelige Balinesen, die sich anmaßten, hochkastige Frauen zu heiraten, hatten früher die Kapitalstrafe zu gewärtigen. Wenn auch adatbedachte Balinesen zu bedenken geben, daß arrangierte Heiraten weniger aus Gründen der Allianzbildung zwischen verwandten bzw. befreundeten Familien erfolgen würden, sondern vielmehr in der Absicht, die etablierte soziale und - damit verbunden - die kosmische Hierarchie zu wahren (BOON 1976: 192), verweisen andere Inselbewohner doch ebenso auf damit verknüpfte handfeste Vorteile. Falls nämlich eine Tochter in eine fremde Brautnehmerfamilie einheiratet, verläßt sie nicht nur ihre Herkunftsfamilie und gibt die Zugehörigkeit zu deren Ahnengruppe auf, sondern sie muß ebenso auf elterliche Erbanteile verzichten. Drastisch kommt dies im balinesischen Sprichwort zum Ausdruck, das eine (ihr Nest verlassende) Krähe ihre Eier aufgebe ("goak ngaluhin taluh»). Sofern nicht im Verband der Großfamilie heiratend, bedeutet die Eheschließung einen entscheidenden Einschnitt in das Leben einer Balinesin, nämlich die Ablösung aus ihrem Familienverband. Deshalb bedürfen die vorausgegangenen Vermählungsfeiern - denen die Brauteltern fernbleiben mußten - einer Abschlußzeremonie (majauman), bei der die Neuvermählten formell den Familienhof und den Ahnenschrein der Brauteltern besuchen, deren Segen erbeten und bei der die Tochter aus ihrem Tempelverband verabschiedet wird (RAMSEYER 1985: 208). 


\subsubsection{Feier zur Schwangerschafi}

Adatgemäß sollte diese Zeremonie zwischen dem sechsten Monat der Schwangerschaft und der Geburt stattfinden. Die Festlegung auf diesen Zeitabschnitt trägt der balinesischen Sicht der Entwicklung der befruchteten Eizelle zum Embryo und zum Fötus Rechnung: Sechs balinesische Monate (zu 35 Tagen) nach der Empfängnis habe der Fötus bereits die Gestalt eines - wenn auch noch sehr kleinen - Menschen angenommen, dessen Körper nun rasch größer und dessen Formen immer prägnanter würden.

Gemäß balinesischer Auffassung wird jeder Mensch von den Vier Geschwistern (kanda mpat; syn. catur sanak)begleitet, im Falle von Männern von vier Brüdern, im Falle von Frauen von vier Schwestern. Sie verkörpern Kräfte, die in magischer Beziehung zum Menschen stehen und ihn nicht nur zu Lebzeiten, sondern auch nach dem Tod begleiten. Je nach Behandlung, die der Betreffende seinen Vier Geschwistern zukommen läßt, können sie ihm Nutzen bringen oder Schaden stiften (HOOYKAAS 1974: 45, 98, 124; WECK 1976: 52-62). In ersterem Fall werden sie als Manifestationen Gottes perzeptiert, in letzterem nehmen die kanda mpat Gestalt von Dämonen an. Je nach ihrem Charakter und je nach Lebensabschnitt ändern sie ihre Namen und ihren Sitz im Körper. Die Herleitung dieser komplexen und oft auch widersprüchlichen Zuordnungen fußt auf Informationen von IDA WAYAN KOMPIANG (dem späteren IDA PADANDA MANUABA) aus Budakeling, ergänzt durch Übersetzungen und Analysen relevanter Lontartexte der beiden Forscher HOOYKAAS (1974) und WECK (1976).

Ausgehend vom Gedanken, daß die Geschwister bereits in den Spermien des Vaters bzw. den Eizellen der Mutter vorhanden sind, werden die Samenwächter väterlicherseits als Geschmack und Farbe, diejenigen mütterlicherseits als Geruch und Gestalt betrachtet. Nach deren Verschmelzung im Eileiter und der anschließenden Fixierung in der Gebärmutter umsorgen die folgenden Vier Mütterlichen Helferinnen das Embryo:

Babu Kakered<smiles>[CH]C</smiles>

Babu Ugian ——

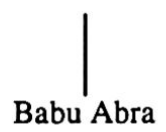

Fig. 7 Die Vier Mütterlichen Helferinnen (babu) des Embryos.

* I Rare Banah = "Nabelschnurkind"; andere Benennung: I Legeprana.
Plazenta (ari-ari)

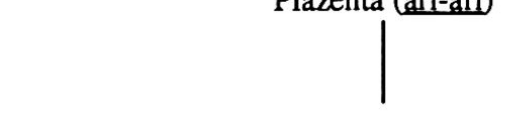

Nabelschnur* _ _umpuking ati —— Fruchtwasser
(banah)

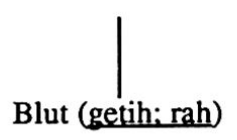

Fig. 8 Die Vier Geschwister des Fötus.

* Anstelle der Nabelschnur werden auch vernix caseosa und/oder Eihäute (lamas bzw. lamad) genannt.

Dabei postulieren Balinesen die Plazenta und das Fruchtwasser als vom Vater, das Blut und die Nabelschnur als von der Mutter stammend. Diese Deutung stimmt mit der uranischen Wertung von Männern (bezüglich Makrokosmos: Richtung Berg bzw. Sonnenaufgang) und der chthonischen Einstufung von Frauen (Richtung Meer bzw. Sonnenuntergang) überein.
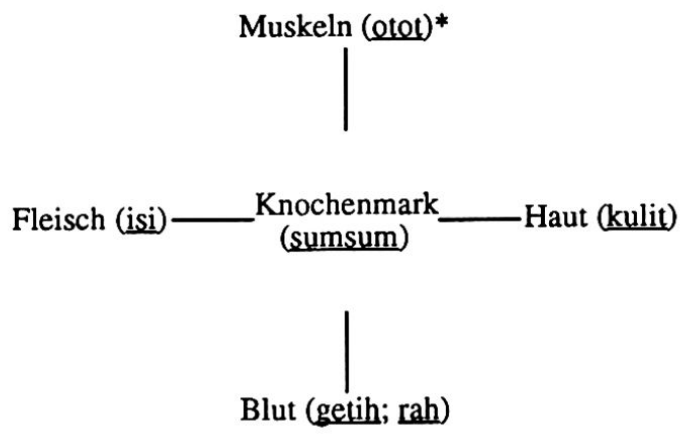

Fig. 9 Die Vier Geschwister bei vollendeter Reife des Fötus (gemäß IDA WAYAN KOMPIANG).

*Anstelle der Muskeln postulieren andere Quellen die Adern.

Schwarzer Buta (b.ireng)

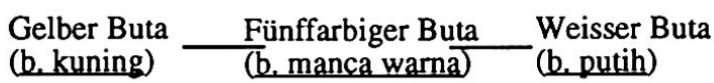<smiles></smiles>

Fig. 10 Die Vier Dämonischen Geschwister des Menschen. 
Die Vier Göttlichen Geschwister des Menschen entsprechen den in Fig. 1 festgehaltenen panca dewata.

Gaben während der Schwangerschaft werden sowohl der werdenden Mutter als auch dem Fötus zugesprochen. So umfaßt das idam-idaman ein Gericht unreifer, saurer Früchte an pikanter Sauce (gelegentlich auch sehr süße Speisen), nach denen einer in der Frühphase Schwangeren besonders gelüstet. Beim madegong-gedongan ${ }^{5}$ gelten Gebete und upakara in erster Linie dem Fötus, verbunden mit der Hoffnung, daß der Nachkomme zu einem würdigen Glied der Gesellschaft heranwachse und die Erwartungen seiner Eltern erfülle. Da im Gedankengut der Balinesen sich der Lebenswandel der werdenden Mutter im Charakter ihres Kindes niederschlägt, wird sie in dieser Phase angehalten, streng darauf zu achten, schlechte Gedanken, Worte und Taten zu meiden und sich an beispielhaften Vorbildern zu orientieren. In adeligen Kreisen wird die Schwangere ermuntert, Geschichten aus dem Mahabharata zu lauschen, in der Hoffnung, daß sich der edle Charakter der Helden auf ihre Kind übertrage. Dem Vater wird geraten, besonders während dieser Phase auf Eintracht in der Familie bedacht zu sein.

Bei Zeremonien Hochadeliger leitet ein mit der Familie verbundener Brahmanenpriester das gottbezogene Ritual im Familientempel der Feiernden. Der padanda segnet das Paar und weiht die für die nachfolgenden Handlungen nötigen Utensilien. In Karangasem werden vor der Eingangspforte zum Tempelgeviert dreimal zwei Zweige des Erythrina-Baumes (dadap) ${ }^{6}$ in den Boden gesteckt und in Brusthöhe mit Fäden verbunden. An den oberen Zweigenden werden mit Wasser gefüllte Blätterbecher befestigt, in denen Fischchen oder Krebschen schwimmen. Die Schwangere hat vorerst dreimal in gebückter Haltung unter den gespannten Fäden hindurchzugehen, Symbol dafür, daß Mutter und Kind willens sind, den Drei Lehrern der Weisheit (triguru wisesa): Eltern, Lehrern und dem Herrscher (bzw. der Regierung) Respekt zu zollen. Beim vierten Durchgang hat die diesmal aufrecht Gehende mit ihrem Oberkörper die Fäden zu zerreißen: Wenn immer im Leben Eltern und Kindern sich Hindernisse in den Weg stellen mögen, sollen sie zielstrebig aus dem Weg geräumt werden. In der Folge schlitzt der Vater mit einem zugespitzten Bambusstecken die Blätterbecher auf, deren Wasser mit den darin enthaltenen Tierchen auf den Boden platscht: So leicht wie Wasser und Fischchen ihren Behälter, so mühelos sollen bei der Geburt Fruchtwasser und Baby den Mutterleib verlassen. Ähnliches Gedankengut kommt darin zum Ausdruck, $\mathrm{da} ß$ die zuvor den buta kala präsentierten Opfergaben so in den Fluß geworfen werden, daß sie «kopfvoran» weggeschwemmt werden, in der Lage also, in der eine Normalgeburt stattfindet.

\subsubsection{Geburt}

Noch vor der Geburt wechselt die Hochschwangere vom Pavillon, den sie normalerweise mit ihrem Mann teilt, in das "Haus der Unreinheit», so beispielsweise in das chthonisch perzeptierte "Haus im Westen» (bale dauh), wo sie - getrennt von ihrem Ehepartner - bis zum 42. Tag nach der Geburt verweilen muß. Wohl in keiner anderen Zeremonie kommt die Bedeutung der Vier Geschwister derart ausgeprägt zum Ausdruck. Balinesen erzählen, daß sich ein Kind im Mutterleib so wohlgeborgen und glücklich fühle, daß es gar kein Verlangen spüre, diesen zu verlassen. Dessen sei sich der Gott der Kleinkinder (Sang Hyang Rare Kumara) bewußt geworden und hätte deshalb dem Säugling versprochen, ihm vier Begleiter zur Seite zu stellen: Beim Eintritt ins Leben würde ihm das Fruchtwasser die Türe öffnen, Blut und Fettschicht (vernix caseosa) würden ihn zur Linken und Rechten schützen, und die Plazenta würde "von hinten stoßen" (ANANDAKUSUMa 1986: $25 \mathrm{f}$.). Unter diesen Vier Geschwistern ist die Nachgeburt das bedeutendste und ersetzt in vielen Gedenkfeiern stellvertretend auch die drei anderen. Nach der Geburt wird die Plazenta sorgfältig gewaschen, in wohlriechendem Wasser gespült und zusammen mit einem Blumengebinde und chinesischen Lochmünzen als Ritualgeld sowie Fasern der Arenga saccharifera in ein weißes Tuch gehüllt und in eine zweigeteilte Kokosnuß gelegt. Mittels magischer Formeln werden die Vier Geschwister beschworen, ihren Platz zu wechseln, vom Fruchtwasser in die Galle, von der vernix caseosa ins Herz, vom Blut in die Leber und vom Mutterkuchen in die Milz. Mitsamt Inhalt wird die Kokosnuß vor der Türe zum Haus der Geburt begraben: links davon, wenn das Neugeborene ein Mädchen ist, rechts davon im Falle eines Knaben. So wird die Plazenta als Ausdruck des Mikrokosmos dem Makrokosmos zurückerstattet.? Über das Plazentragrab werden sauber geschrubbte, kopfgroße, schwarze Steine gelegt, damit der beerdigte Mutterkuchen von Störungen verschont bleibe. Pragmatisch urteilende Balinesen rechtfertigen diesen Grabschutz damit, daß so streunende Hunde und Schweine nicht an die Plazenta gelangen könnten. Neben den Steinen in den Boden gesteckte stachelige Pandanblätter dienen der Abwehr schwarzmagischer Einflüsse, welche die körperlich als unrein gewertete Mutter zu bedrohen trachten. Kokosfasermäntel und Weihrauchstäbchen werden angezündet, um mit dem Rauch blutrünstige Dämonen abzulenken. Ähnliches wird mit einem Gewürzwasser enthaltenden Bananenblattbecher angestrebt, mit dessen Wasser die durch Blut und Nachgeburt befleckten Hände gewaschen werden. Aufschlußreich ist unter anderen ein Präsent, bestehend aus Ingredienzien eines vollständigen Betelbissens: Sirihblätter (Piper betle), Betelnuß (Areca catechu), Gambir (Uncaria gambir), Kalk und Kautabak. Diese Gabe soll gemäß Information meiner Gastgeber den im Neugeborenen inkarnierten Ahnen willkommen heißen.

\subsubsection{Nabelschnurabfall}

Nach balinesischer Auffassung ist am Tag des Nabelschnurabfalls die Plazenta bereits verwest. Deshalb stehe nun die Nabelschnur als letzter materiell existierender Vertreter der Vier Geschwister im Zentrum des Gesche- 
hens. Zusammen mit scharfen Gewürzen wird sie in ein neues weißes Tuch gewickelt und anschließend über dem Schlafplatz des Säuglings in der uranisch gewerteten Richtung bergwärts (kaja) aufgehängt. Da die Mutter noch immer nicht frei ist von "unreinem Ausfluß», müssen Dämonen (leak) vom fatalen Geruch abgelenkt werden. Dies geschieht nicht zuletzt mit einem Amulett, das stark riechende Gewürze (so u. a. Knoblauch) enthält und von Mutter und Kind bis zum 42. Tag nach der Geburt in einem Armband aus Garn um das rechte Handgelenk getragen wird. Gleichzeitig soll der Talisman vor Krankheiten jeglicher Art schützen. Auf dem Opferaltar über der Wiege des Säuglings werden bis zu dessen erstem Geburtstag (d. h. bis zum 210. Tag nach der Geburt) täglich kleine Gaben dargereicht. Über dem Plazentagrab ist ein Bambusaltar (sanggah cucuk) errichtet worden, auf dessen Plattform auch die Vier Geschwister ihren Anteil an der Säuglingsnahrung (banten jotang, so z. B. einige Tropfen Muttermilch) erhalten. Gemäß ANANDAKUSUMA (1986: 14) wechseln nun die kanda mpat ihre Namen wie folgt:

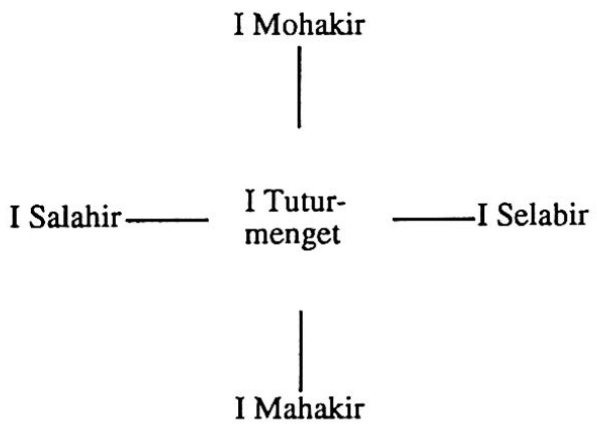

Fig. 11 Die Vier Geschwister am Tag des Nabelschnurabfalls.

\subsubsection{Zwölftagefeier}

Mit der Überreichung von Präsenten an den Gott der Kleinkinder bitten die Eltern für ihr Neugeborenes um Wohlfahrt, langes Leben und um den Schutz des Allmächtigen. In Karangasem sollen weitere kleine upakara Gott gütig stimmen und es der Mutter erlauben, die "zwölftägige Schwelle ihres Leidens» zu überschreiten. Dieser zeremonielle Akt entspricht gleichzeitig einer ersten - wenn auch noch nicht definitiven - Purifikation der Mutter. Gelegentlich ziehen Feiernde einen balian bei, dem die Fähigkeit zugeschrieben wird, sich mit Verstorbenen in Verbindung setzen zu können. Er wird gebeten, den Ahnen zu benennen, der im Kleinkind inkarnierte. Dabei ist es nicht unüblich, daß ein und derselbe Vorfahre als bereits in anderen Kindern wiedergeboren betrachtet wird. Nachdem dem Baby bei seiner Geburt automatisch der Geschlechtsindikator und die Geburtsfolgebezeichnung zugesprochen worden sind, erhält der Säugling an seinem zwölften Lebenstag seinen Eigennamen, den er bis zur Mutter- bzw. Vaterwerdung beibehält und dann erneut ändert. ${ }^{8}$

\subsubsection{2-Tage-Feier}

42 Tage nach der Geburt hat die Zeitspanne der durch das Gebären bedingten körperlichen Unreinheit der Mutter ein Ende gefunden. Denn die Zeit der Enthaltung bzw. Schonung (kakambuhan) ist nun vorüber, und die Frau darf das Haus der Entbindung verlassen und wieder mit ihrem Mann zusammen wohnen. Da nun keine Gefahr mehr besteht, daß blutgierige Dämonen die Mutter heimsuchen, kann das Amulett zur Abwehr der Vampire entfernt werden (megat kakambuh: "das Amulett aufschneiden"). Nach absolviertem Reinigungsritual ist es ihr erlaubt, wiederum die Küche und den Familientempel zu betreten sowie an allen Aktivitäten der Dorfgemeinschaft teilzunehmen. In der Küche erhält Batara Brahma rotfarbene Opfergaben. Am Badeplatz, wo sich die bis anhin unreine Mutter zu waschen pflegte, wird Batara Wisnu (der u. a. auch über das Wasser gebietet) beschenkt und im Familientempel Batara Guru bedacht. Gemäß adat von Karangasem wird der Säugling an diesem Tag erstmals nach seiner Geburt gebadet und somit die Voraussetzung zu dessen Teilnahme an gemeinsamen Gebeten im Familientempel und zum Empfangen von Weihwasser geschaffen.

Nebst dem angesprochenen Purifikationscharakter für Mutter und Kind nimmt die Feier aber auch Bezug auf die Verabschiedung von den 108 Kräften (nyama bajang), welche zuvor den Vier Geschwistern geholfen haben, den Fötus im Mutterleib heranreifen zu lassen, nach der Geburt nun aber nicht mehr als Beschützer auftreten, sondern im Gegenteil das Wohlbefinden des Neugeborenen gefährden. Um die bajang an ihren Ursprungsort zurückzuweisen, werden sie mit banten pacolongan bedacht. Auffallend ist dabei vor allem eine Gabe, die als Abgeltung für bajang colong bestimmt ist, nämlich zwei Küken (je ein weibliches und ein männliches), von denen gewissenorts angenommen wird, daß sich der genannte bajang mit diesem Präsent dann besonders zufriedengestellt fühle, wenn die beiden Hühnchen zuvor gestohlen worden sind und ihm dann gebacken als Wegzehrung gereicht werden. Erhofft wird damit, daß einerseits bajang colong und seine Gefährten sich endgültig davonmachen und von nun an das Baby nicht mehr durch ihre Präsenz stören. Andererseits soll der vorangegangene Akt die Begierde zu stehlen im späteren Leben des Kindes dämpfen.

\subsubsection{Dreimonatsfeier}

Drei balinesische Monate zu 35 Tagen nach der Geburt wird der Säugling zum Kind und darf von nun an, getragen von den Eltern (im Falle eines Knaben vom Vater, im 
Falle eines Mädchens von der Mutter), den Familienhof verlassen. Der anläßlich der Zwölftagefeier zugesprochene und bislang in der Familie geheimgehaltene Rufname des Kindes wird jetzt allen Anwesenden offiziell verkündet und somit legitimiert. Von nun an heißen die Vier Geschwister: Banaspati Raja, Anggapati, Marajapati und Banaspati.

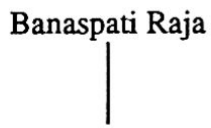

Branaspati —_ Kala Mretiu*__ Anggapati

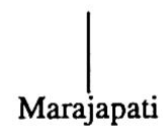

Fig. 12 Pancamahabuta.

* Kala Mretiu $=$ Gott des Todes. Anstelle von Kala Mretiu wird auch Siwa genannt.

Die Pancamahabuta (inkl. Zentrum) machen die Fünf Wesenheiten der Großen Welt aus, wobei hier deutlich wird, daß der genuin balinesischen Vorstellung der kanda mpat nun ein indischer Raster, übernommen aus dem Mahabharata-Epos, zugrunde gelegt worden ist.<smiles>CC(C)(C)[14CH3]</smiles>

Erde (pertiwi) _ Himmel; Raum* $\underset{\text { (akasa) }}{\text { Wind; Luft (bayu) }}$<smiles>CCC(C)C(C)C(C)C</smiles>

Fig. 13 Die Fünf Wesenheiten des Makrokosmos. * Der Raum wird als "göttliche Leere" bezeichnet.

Laut WECK (1976: 62 ff.) fußt die Perzeption aufdem indischen Schöpfungsmythos, der davon ausgeht, daß Sang Hyang Sunia vor der aktiven Phase der Schöpfung vorerst in der Leere die auf dem Lotus schwebende göttliche Einsamkeit (den Raum) schuf, und aus dem Raum der Wind, aus dem Wind das Feuer, aus dem Feuer das Wasser und aus diesem die Erde entstanden sei. Gleich wie alles aus dem Raum abgeleitet werde, könne aber auch alles wieder in die göttliche Leere verschwinden: die Erde im Wasser, das Wasser im Feuer, das Feuer im Wind und letzterer im Raum.

Da der menschliche Körper nicht nur Ebenbild der GroBen Welt, sondern mit ihr eins ist, nehmen die Pancamahabuta auch Besitz vom Menschen und verlassen ihn nach dem Tod wieder. Sie sind seine körperlichen und psychischen Charakteristika, seine Sinne und Leidenschaften.

\section{Makrokosmos}

Raum
Wind; Luft
Feuer; Licht
Wasser
Erde

\section{Mikrokosmos}

\begin{tabular}{|c|c|}
\hline$\langle-\cdots$ & Schädel \\
\hline$<--\cdot-\cdot-\cdot\rangle$ & Atem \\
\hline 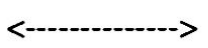 & Augen \\
\hline <-------------> & Zunge \\
\hline$<-$ & Haut \\
\hline
\end{tabular}

Fig. 14 Pancamahabuta im Makrokosmos und im Mikrokosmos (WECK 1976: 64).

Bei der Auflösung des Körpers geht die Haut in die Erde über, die Zunge ins Wasser, die Augen ins Feuer, der Atem in den Wind und der Schädel in den Raum. Wenn die Fünf Wesenheiten des menschlichen Körpes inexistent geworden sind, sind sie Erde, und die Erde verschwindet im Wasser, das Wasser im Feuer, das Feuer im Wind und der Wind im Raum (WECK 1976: 64).

Die Pancamahabuta treten über bestimmte Körperteile in den Menschen ein (1), nehmen dort Sitz (2) und verlassen ihn über andere Organe wieder (3).

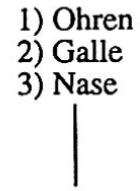
1) Nase
2) Nieren
1) Haarspitzen
3) Augen
2) Herzbeutel
1) Augen
3) Fontanelle
2) Herz

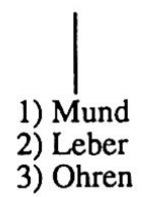

Fig. 15 Eintrittstellen, Sitzplätze und Austrittsorte der Pancamahabuta im menschlichen Körper (gemäß Informationen von I.W.KOMPIANG 1972; HOOYKAAS 1974: 106; WECK 1976: 47, 61). 
Wenn immer möglich, wird zur Durchführung der Dreimonatefeier ein Priester eingeladen, der wirksames Weihwasser zuzubereiten weiß und die Zeremonien leitet. Letztere sind nach den zuvor hauptsächlich körperbezogenen Reinigungen nun in der nyambutin-Feier stärker auf die Seele ausgerichtet. Gewisse Handlungen nehmen Bezug auf den Entwicklungsstand des Kleinkindes, wie z. B. das Sitzenlernen. Beim upacara tuun di pane etwa wird das Kleinkind von der es tragenden Frau dreimal auf eine Tonschüssel (pane) gesetzt, dann in elf Arten von wohlriechenden Blumen enthaltendem Wasser gebadet, wie auch das Haar des Kindes gewaschen wird. Diese Aktivitäten sind in das Zeremoniell des dreimaligen «Umschreitens des steinernen Mörsers» eingebettet, bei dem die Begleitpersonen in Stoffreste gekleidete und mit menschlichen Figuren bemalte "Puppen» aus Kürbis, aus dem Kolben der Bananenblüte, aus Palmblattrippen, Rindenstücken, Eiern, Steinen usw. tragen. Die Figuren haben die Funktion von Wechselbälgen, mit denen potentielle Störefriede vom "echten» Kind abgelenkt werden sollen. Sie werden sogar vor den Priester gebracht und von diesem zum Schein purifiziert.

In einem irdenen Topf, der inmitten eines mit Blumen und Blättern verzierten symbolischen Gärtchens steht, sind Fischchen, Garnelen, Reishalme, aber auch Geldstücke und Schmuck aller Art enthalten. Mit dem, was das Kind mit seinen Händchen zuerst ergreift - so will es das Orakel - wird es zeitlebens gesegnet sein. AnschlieBend wird dem nun mit Schmuck aller Art versehenen Säugling ein geflochtener Hahnenkorb übergestülpt, wo ein Küken den Schmutz von der Stirn des Säuglings pikken soll. Nachdem das gefeierte Kind vor den Priester geführt und mit Weihwasser besprenkelt worden ist, schneidet dieser dem Kind die Haare, streng darauf achtend, daß ein die Fontanelle bedeckender Haarbüschel (jam$b o t)$ übrigbleibt. Wohlhabende Familien kleben gelegentlich auch ein goldenes Medaillon über diese besonders gefährdete Stelle ihres Jüngsten. Bevor die Feiernden nun im Familientempel das Yadnya-Ritual abschließen und sich einen Langgrashalm (Imperata cylindrica) um die Stirn binden, sind die zur Mokierung niederweltlicher Störefriede verwendeten Wechselbälge bereits außerhalb der Mauer des Familienhofes weggeworfen worden.

\subsubsection{Feier des ersten Geburtstags}

Ist das Kind 210 Tage, d.h. gemäß javanisch-balinesischem Kalender ein Jahr alt geworden, findet die erste Geburtstagsfeier statt. Mit der Darreichung von panebusan oton-Gaben erhoffen sich Balinesen die Wiedergutmachung von Fehlern, begangen in den Vorleben, und drücken ihren Wunsch nach Vervollkommnung aus. Im Rahmen dieses Anlasses erfolgt oft der erste vollständige Kahlschnitt der Haare. Er zielt auf die Reinigung der Fontanelle ab. Ist ein erster Haarschnitt bereits während der Dreimonatefeier durchgeführt worden, wird nun auch der jambot entfernt. Drei Tage nach der Zeremonie wird das geschnittene Haar hinter dem Ahnenschrein oder an einem anderen als günstig erachteten Ort vergraben.

Nachdem der Säugling anläßlich der 105-Tage-Zeremonie erstmals auf den pane (siehe oben) gesetzt worden ist, darf das Kind nun zum ersten Mal mit seinen Füßen mit dem Boden in Berührung gelangen. Bis zu diesem Zeitpunkt mußte das Jüngste immer getragen werden, um es als "Ebenbild Gottes» (maraga dewa) nicht mit dem «Schmutz der Erde» in Kontakt kommen zu lassen. Zur Vorbereitung des jetzt zum «vollen Menschen» gewordenen Kindes wird Mutter Erde (Ibu Pertiwi) um Erlaubnis zur Durchführung des Bodenberührrituals gebeten, wirkt diese Göttin doch als Gegenkraft zur an und für sich chthonisch bestimmten Sphäre. Die Stelle, wo die Füße erstmals mit der Erde in Kontakt kommen, wird sorgfältig mit einem Bedawang Nala markiert, d. h. mit einer Fabelgestalt in Form einer mit einem Rüssel versehenen Schildkröte, um die sich zwei mythologische Schlangen winden. Sie symbolisiert das Fundament der Welt und findet sich z. B. auch am Sockel von Tempelschreinen.

\subsubsection{Feier des Enwachsenwerdens}

Mit dieser Zeremonie wird dem Heranwachsenden der Übergang vom Kindesalter zum Erwachsenen vor Augen geführt. Er soll sich bewußt werden, daß er von nun an dem Einfluß von Sang Hyang Semara und Sang Hyang Ratih unterliegt. ${ }^{9}$ Als Zeitpunkt für das "Erwachsenwerden» von Mädchen wird die erste Menstruation, für Knaben (wenn für sie diese Feier überhaupt durchgeführt wird) der Stimmbruch als geeignet erachtet. Die gefeierte Person löst sich vom Kindesdasein, verbringt als Übergangsphase 24 Stunden (früher drei volle Tage) in einem dunklen Raum in Klausur, wird - anschließend ans Licht tretend - in die Welt der Erwachsenen integriert und Schritt um Schritt mit dem geschlechtsspezifischen Rollenverhalten vertraut gemacht. Letzteres beinhaltet beispielsweise die strikte Geschlechtertrennung bei spielerischen Betätigungen, bestimmten Arbeiten und vor allem bei religiösen Verpflichtungen. Als Kinder von ihren Eltern umsorgt und erzogen, beginnen Jugendliche ihr Handeln zunehmend eigenständiger zu bestimmen. Während ihrer bewegten Phase der Verunsicherung und körperlicher Versuchungen benötigen Pubertierende göttlicher Führung, die Semara Ratih ${ }^{9}$ anvertraut wird. Die «Heirat» mit dieser Gottheit fuihrt zu neuen Erfahrungshorizonten. Gebete an Semara Ratih und an die Widiadara und Widiadari (männliche bzw. weibliche Nymphen als Begleiter von Semara Ratih) sind mit der Bitte verbunden, daß göttliche Kräfte die körperlichen Begierden ausbalancieren mögen, die Person aber gleichzeitig begehrenswert und in der Liebe glücklich werden lassen.

$\mathrm{Da} ß$ sich die Feier vor allem an Töchter richtet, ist weniger der Tatsache zuzuschreiben, daß sich bei diesen der "Zeitpunkt des Erwachsenwerdens» (menek kelih) eindeutiger als bei Knaben bestimmen läßt, als vielmehr mit 
der Wertvorstellung verknüpft, daß das moralische Verhalten einer Frau das Image der Familie stärker beeinflusse als das Verhalten eines Mannes. ${ }^{10}$ PUTRA (1974/2: 42 f.) begründet die Wertung der Frau als Gradmesser für das Ansehen einer Familie mit Versen aus den im Mahabharata enthaltenen Bhagwad Gita:

"Wenn eine Familie am Zerfallen ist, stirbt ihre Moral: wenn ihre Moral stirbt,

beherrscht das Gefühl von Amoral die Familie. Und wenn Amoral die Situation bestimmt, o Krisna, erfahren die Frauen der Familie einen moralischen Niedergang;

und wenn die Moral der Frauen zusammenbricht, o Krisna,

so resultiert daraus Chaos in der Menschheit!»

Im Gebäude, in dem die erstmals Menstruierende verweilt, steht eine Bettstätte, ausgerüstet mit Matratze, Kissen, Bettüchern und einer Schlafmatte, versehen mit einer Zeichnung von Semara Ratih. Die Opfergaben, u. a. in weißer und gelber Farbe" für die Gottheit der Liebe und ihre Nymphen (banten padadarian), werden auf das Kopfende des Bettes gelegt. Gelb sind auch die Bettvorhänge, das Flechtwerk aus jungen Kokosblättern, die Blumenbouquets, die dargereichten neuen Textilien wie auch die Stoffe, in die sich die Gefeierte hüllt. Am Badeplatz ist ein Bambusaltar errichtet worden. Bereitgestelltes Wasser enthält wohlriechende Blütenblätter und ist ausschließlich für die Menstruierende bestimmt. In Sanur wird das festlich gekleidete Mädchen zum Schluß auf einer Sänfte zum Priester getragen, der das anschließende Ritual für die versammelte Familie leitet.

\subsubsection{Zahnfeilzeremonie}

Frauen werden die Zähne in der Zeitspanne zwischen erster Monatsblutung und der Heirat, Männern zwischen dem 16. Altersjahr und dem Tod, im Extremfall sogar erst nach ihrem Ableben, gefeilt. ${ }^{12}$ Fromme Balinesen freilich betonen, daß das Zahnfeilen Dahingeschiedener nicht adatkonform sei. Bearbeitet werden die obern, d. h. uranisch gewerteten, vier Schneidezähne und die zwei Eckzähne, während die entsprechenden Zähne des chthonisch bestimmten Unterkiefers in ihrem ursprünglichen Zustand belassen werden. Äußerlich betrachtet, mögen in dieser Zeremonie Elemente zur Steigerung der Anmut und der Attraktivität wahrgenommen werden, widersprechen doch lange und scharfe Zähne dem Schönheitsideal der Balinesen. Doch die Zeremonie beinhaltet mehr, sind doch die sechs erwähnten Zähne Symbol der "Sechs Feinde (und Verführer) des Menschen» (sadripu). Gemäß Auffassung balinesischer Hinduisten wird der Lebenswandel durch das Wechselspiel gegensätzlicher Charaktereigenschaften bestimmt, so einerseits durch guna satwam, d. h. durch den Ruhe und Gelassenheit ausströmenden Pol der Güte, Aufrichtigkeit, Edelmut und Abgeklärtheit. Andererseits unterliegt menschliches
Handeln den guna rajas tamas, wobei guna rajas dynamische Charakterzüge wie Leidenschaft, Ehrgeiz usw. umfassen und guna tamas aktivitätshemmende Elemente, so Passivität, Faulheit, Gleichgültigkeit usw., beinhalten. Falls sich der Mensch auschließlich von guna rajas tamas leiten läßt, unterscheiden sich seine Charakterzüge kaum von denjenigen von Tieren oder niederweltlichen Wesen. Er kann sich von ihnen nur dann abheben, wenn er nebst dem Verlangen nach irdischen Gütern und Lebensfreude auch nach tugendsamem Lebenswandel strebt, m. a. W. auf ein sorgfältiges Ausbalancieren von uranischen und chthonischen Einflüssen bedacht ist.

Als die sechs Feinde des Menschen werden genannt: sinnliche Begierde (kama), Jähzorn (krodha), Habgier (lobha), Rausch (moha), Arroganz (mada) und Mißgunst (matsarya). Sie gilt es, in der Zahnfeilzeremonie symbolisch zurückzustutzen und durch die Belassung der unteren Zähne in ihrem ursprünglichen Zustand die Ausgewogenheit zwischen aktivem, zielstrebigem Handeln einerseits und Besonnenheit andererseits im Sinne von mens sana in corpore sano zu versinnbildlichen.

Anläßlich der Zahnfeilung erhält die Gottheit leidenschaftlicher Liebe ein gelbes Bambushäuschen (bale gading) als Sitzplatz zugesprochen. Es ist überdacht mit goldgelben Blumen, wie auch die Verzierungen und die dargereichten Stoffe von gelber Farbe sein sollten. Die Liegestätte ist reich geschmückt, wobei Bilder von Semara Ratih den Ort des Zeremoniells zieren. Die Opfergaben ähneln denjenigen im bale gading und sind der genannten Gottheit und den diese begleitenden Nymphen zugedacht. $\mathrm{Zu}$ den für den eigentlichen Feilprozeß bereitgestellten Utensilien gehört u. a. eine junge, mit dem Semara Ratih-Symbol versehene gelbe Kokosnuß, deren Fruchtwasser zuvor ausgeleert worden ist. Später als Spucknapf benutzt, wird sie auf ein - wenn möglich - silbernes Tablar gelegt, auf dem sich auch auf 1 bis $1 \frac{1}{2} \mathrm{~cm}$ Länge zugeschnittene Klötzchen aus einem Zuckerrohrstengel und einem entrindeten Erythrina-Zweig befinden. Sie werden in der Folge zwischen Ober- und Unterkiefer geklemmt (rechts bei einem Mann, links bei einer Frau), damit der Feilungskandidat während der Prozedur nicht versehentlich den Mund schließt, dabei den feilenden Priester beißt und die sadripu auf ihn überträgt. Ferner befinden sich ein Fingerring mit einem Rubin, ein Spiegel, ein Hämmerchen, ein Taschentuch zum Abwischen des Mundes sowie ein vollständiger Betelbissen und eine Betelnußschere (caket) auf dem Tablar. Feilen und Meißel werden in einer mit Wasser gefüllten Schale aufbewahrt.

Nach der Zufriedenstellung der chthonischen Kräfte durch caru-Gaben setzt sich der zu Feilende vorerst auf die oben genannte Liegestätte. Die Gebete sind an Sang Hyang Semara Ratih gerichtet. Darauf nimmt der Priester den Fingerring mit dem Rubin in seine rechte Hand und "zeichnet" damit magische Symbole über den Körper des Kandidaten. Nachdem letzterer die Essenz der Opfergaben gegen sich zugefächelt hat (natab) und mit Weihwasser besprenkelt worden ist, legt er sich auf die 
mit einer Schlafmatte bedeckte Matratze, bettet seinen Kopf auf das Richtung bergwärts oder Osten liegende Kissen, bedeckt seinen Körper mit einem gelben Tuch und faltet seine Hände über der Brust. Die eigentliche Feilprozedur beginnt erst dann, wenn ein Bamubskeil zwischen Ober- und Unterkiefer getrieben worden ist und der Zeremonienleiter die Zähne mit Hammer und Meißel «abgetötet» hat ( matatah $=$ abmeißeln). Jetzt wird der Schmelz der oberen sechs Zähne (von Eckzahn zu Eckzahn) ebenmäßig gefeilt. Dicht gedrängt umstehen Familienangehörige und Befreundete die Liegestätte. Während der schmerzhaften Prozedur halten Danebenstehende die Hände des Gefeilten, um während dieser Phase der Verwundbarkeit Böses abzuwenden. An der Feier teilnehmende alte Männer mögen die zeremonielle Handlung mit Singen balinesischer Fabeln begleiten. Von Zeit zu Zeit überprüfen Gefeilte die ästhetische Wirkung im Spiegel und flüstern dem Ausführenden Anweisungen zu. Bei Unterbrüchen im Feilprozeß wird der zum Offenhalten des Mundes verwendete Bambuskeil (zusammen mit dem Speichelfluß) in die bereitgestellte Kokosnuß gespuckt und durch ein Stück aus dadap-Holz ersetzt. Erst nach der "Wiederbelebung» der Zähne durch Sprechen einer magischen Formel darf sich die Person erheben und mit ihren Füßen die den Dämonen gewidmeten Gaben berühren. Sie gurgelt mit aromatischem Wasser und kaut den Betelbissen. Letzteres soll die Zähne stärken und Mundgeruch vertreiben. Die Zeremonie endet mit Gebeten im Familientempel. Gefaßter sehen Gefeilte nun der Zeit der Anfechtungen entgegen, dürfen sie doch hoffen, durch Einbezug von guna satwam ihre Leidenschaften besser zügeln zu können.

\section{Kurzer Hinweis auf weiterführende Zeremonien}

Mit dem Tod fällt der Mensch den Mächten der Niederwelt anheim. Körper und Seele des Dahingeschiedenen sind dann in höchstem Maß unrein und bedürfen zahlreicher ritueller Purifikationen (pitra yadnya). Erst nach der Kremation des Leichnams und den anschließenden Läuterungen der Seele kann diese schrittweise von ihrer Bindung an die Welt gelöst und schließlich zum vergotteten Ahnen (Sang Dewa Yang) werden, der sich im Ahnenschrein (kemulan) seitens seiner Nachkommen göttlicher Verehrung erfreut.

\section{Anmerkungen}

Der vorliegende Aufsatz beruht einerseits auf der Auswertung themenrelevanter Literatur, verfaßt von balinesischen Autoren und westlichen Forschern (vgl. Literaturverzeichnis), und andererseits auf persönlichen Beobachtungen und Auswertungen meiner Bilddokumentation, ergänzt durch zahlreiche Hinweise balinesischer Freunde und Informanten. Die Orthographie balinesischer Wörter und Begriffe aus dem Sanskrit folgt weitgehend dem Kamus Bali-Indonesia (Denpasar, 1978).
1 Lontargelehrte beziehen ihr Wissen aus klassischen, meist in altbalinesischer oder altjavanischer Sprache abgefaßten Texten, die auf getrockneten Blättern der Lontarpalme (Borassus flabellifer) eingeritzt worden sind und immer und immer wieder kopiert werden.

${ }^{2}$ Das adat gilt als intellektuelles Vermächtnis der Ahnen und ist sozialer Ausdruck der Gemeinschaftsreligion. Somit wird es nicht als ein Werk von Menschen wahrgenommen. Zur umfassenden Begriffsbedeutung vgl. ALISJAHBANA, 1966: 4-6.

${ }^{3}$ Aufgrund der exzessiven Verwendung von Weihwasser wird der balinesische Hinduglaube oft als "Weihwasserreligion" (agama tirta) bezeichnet.

${ }^{4}$ Zu beachten ist, daß gewisse Feiern - nicht zuletzt aus Kostengründen - über das korrekte Datum hinaus verschoben und mit anderen rites de passagezusammengelegt oder gemeinsam mit weiteren Personen aus der Großfamilie oder der Dorfgemeinschaft durchgeführt werden. Zudem fallen die genannten Feiern aufgrund lokal variierender Adatbestimmungen nicht immer auf den im Text erwähnten Zeitpunkt wie auch einzelne symbolische Handlungen verschiedene Ausdrucksformen aufweisen können.

${ }^{5}$ GemäßMERSHON (1971:51) ist das balinesische Verb magedong-gedongan mit "das Haus füllen" zu übersetzen. Im übertragenen Sinn wird bei der Schwangerschaftsfeier das "Füllen des mütterlichen Bauches" angesprochen. Je nach Lontarquelle sind Variationen der Namen und Anordnungen in den erwähnten Vierer- bzw. Fünfersystemen möglich.

${ }^{6}$ Der dadap-Baum ist ein Symbol der Lebenskraft. Denn wie alt bzw. dürr ein Erythrina-Zweig auch immer sein mag schlagen nach seinem Einstecken in den Boden rasch wieder Blätter aus.

${ }^{7}$ Während Plazentabeisetzungen auch bei anderen Ethnien stattfinden, wie z. B. bei den Ifugao im Bergland von Luzon (Philippinen), scheinen die restlichen "Geschwister" auf Bali beschränkt zu sein und autochthonem Gedankengut zu entspringen.

${ }^{8}$ Geschlechtsindikatoren für nichtadelige Balinesen: I, für Balinesinnen : Ni.

Geburtsfolgebezeichnungen für Balinesen und Balinesinnen:

1. (bzw. 5., 9.) Geborenes ein und derselben Mutter: Wayan (oder Putu)

2. (bzw. 6., 10.)

3. (bzw. 7., 11.) Made (oder Nengah) Nyoman

4. (bzw. 8., 12.) Ketut.

Zur balinesischen Teknonymie siehe GEERTZ und GEERTZ, 1975: 85-94, 199.

9 Sang Hyang Semara Ratih ist die Gottheit der Anmut, Liebe und der Leidenschaften. Sie umfaßt die männliche Komponente Sang Hyang Semara (Sonnengott) und dessen weibliche Komplementarität Sang Hyang Ratih (die «bildschöne Mondgöttin"). So ist beispielsweise die balinesische Redewendung "sampun kaanan Semara Ratih" mit "bereits das Gefühl der Liebe kennen" zu übersetzen. Bisexuelle Erscheinungsformen werden unter dem Begriff arda nareswaribzw. purusa pradana subsumiert ( purusa $=$ männlich; pradana $=$ weiblich).

${ }^{10}$ Diese Wertung kommt beispielsweise auch in der javanischen Gesellschaft ausgeprägt zur Geltung.

"Weiß und gelb sind nicht nur Symbolfarben für "Osten" bzw. "Westen" und deren Richtungswächter /swara bzw. Mahadewa (vgl. Fig. 1, 4), sondern auch für Semara Ratih. Dabei wird Semara als männlicher Komponente weiß, Ratih als weiblicher Komponente gelb zugesprochen. 
${ }^{12}$ Ist dies möglichenweise ein weiterer Hinweis darauf, daß das Image einer Familie stärker vom moralischen Verhalten der Frau als von demjenigen des Mannes abhängt?

\section{Literatur}

ALISJAHBANA, S. T. (1966): Indonesia: Social and Cultural Revolution, Kuala Lumpur, Singapore, London, Melbourne.

ANANDAKUSUMA, Sri Rsi I G. (1972): Manusa Yadnya, Pitra Yadnya dan Kidung Yadnya, Klungkung (mimeo).

ANANDAKUSUMA, Sri Reshi (1986): Aum Upacara Manusa Yadnya, Denpasar.

BIASIO, E., MÜNZER, V. (1980): Übergänge im menschlichen Leben. Geburt, Initiation, Hochzeit und Tod in außereuropäischen Gesellschaften, Zürich.

BOON, J.A. (1977): The Anthropological Romance of Bali, 1597-1972, Cambridge, London, New York, Melbourne.

EISEMAN, F. B. Jr. (1989): Bali, Sekala and Niskala, vol. I: Essays on religion, ritual, and art, Berkeley, Singapore.

GEERTZ, H., GEERTZ, C. (1975): Kinship in Bali, Chicago, London.

van GENNEP, A. (1981): Übergangsriten (Les rites de passage), Frankfurt, New York, Paris.

HOOYKAAS, C. (1974): Cosmogony and Creation in Balinese Tradition, The Hague.

LEEMANN, A. (1976): Bali. Auswirkungen des balinesischen Weltbildes auf verschiedene Aspekte der Kulturlandschaft und auf die Wertung des Jahresablaufes. In: Ethnologische Zeitschrift Zürich, II, 27-65.
LEEMANN, A. (1979): Bali, Innsbruck, Frankfurt a. M.

MERSHON, K. (1977): Seven plus seven. Mysterious Life-Rituals in Bali, New York, Washington, Hollywood.

MOERDOWO, R. (1973): Ceremonies in Bali, Yogyakarta, Jakarta.

PANITIA PENYUSUN KAMUS BALI-INDONESIA (1978): Kamus Bali-Indonesia, Denpasar.

PUTRA, I G. Agung (?): Upatjara Potong Gigi, Denpasar (mimeo).

PUTRA, I G. Agung (1974/1): Cudamani. Kumpulan Kuliah² Agama, jilid I, Denpasar (mimeo).

PUTRA, I G. Agung Mas (1974/2): Upacara Manusa Yadnya, Denpasar.

PUTRA, I G. Agung Mas (?): Upakara-Yadnya, Denpasar.

PUTRA, I G. Agung Mas (1987): Upacara Manusa Yadnya, cetakan III, Jakarta.

PUTRA, I G. Agung Mas Muterini (1988): Panca Yadnya, Jakarta.

RAMSEYER, U. (1977): Kultur und Volkskunst in Bali, Fribourg.

RAMSEYER, U. (1985): Brauterwerb und Hochzeit in der Hindu-Balinesischen Religion. In: Die Braut. Geliebt, verkauft, getauscht, geraubt. Zur Rolle der Frau im Kulturvergleich, Bd.1, Köln 202-209.

STUART-FOX, D. J. (1974): The Art of the Balinese Offering, Yogyakarta.

WECK, W. (1976): Heilkunde und Volkstum auf Bali, Jakarta. 